\title{
Nonholonomic Control Systems: From Steering to Stabilization with Sinusoids *
}

\author{
Andrew R. Teel \\ EECS Department \\ University of California \\ Berkeley, CA 94720
}

\author{
Richard M. Murray \\ Mechanical Engineering \\ California Institute of Technology \\ Pasadena, Ca 91125
}

\author{
Greg Walsh \\ EECS Department \\ University of California \\ Berkeley, CA 94720
}

\begin{abstract}
In this paper we present a control law for globally asymptotically stabilizing a class of controllable nonlinear systems without drift. The control law combines earlier work in steering nonholonomic systems us ing sinusoids at integrally related frequencies, with the ideas in recent results on globally stabilizing linear and nonlinear systems through the use of saturation functions. Simulation results for stabilizing a simple kinematic model of an automobile are included.
\end{abstract}

\section{Introduction}

This paper focuses on the problem of point stabilization for a control system of the form

$$
\dot{x}=\sum_{i=1}^{m} g_{i}(x) u_{i} \quad x \in \mathbf{R}^{n},
$$

where each $g_{i}$ is a smooth vector field on $\mathbf{R}^{n}$ and the $g_{i}$ 's are linearly independent for all $x \in \mathbf{R}^{n}$. Systems of this form arise in the study of mechanical systems with velocity constraints and have received renewed attention as an example of strongly nonlinear systems. For such systems, control methods based on linearization cannot be applied and nonlinear techniques must be utilized. We are particularly interested in the case where the nonlinear system (1) is completely controllable, corresponding to a set of maximally nonholonomic constraints which do not restrict the state of the system to a submanifold of the state space. See [12] for a more detailed derivation and motivation. We refer to a system with these properties as a nonholonomic control system.

A fundamental problem in the study of nonholonomic control systems is the generation of open-loop trajectories connecting two states. That is, given an initial state $x_{0}$ and a final state $x_{1}$, find an input $u(t)$, $t \in[0,1]$ such that $x(0)=x_{0}$ and $x(1)=x_{1}$. Such an input induces a feasible state trajectory which automatically satisfies the constraints on the system. The condition for the existence of a path between two configurations is given by Chow's theorem. We let $[f, g]$ be the Lie bracket between two vector fields.

$$
[f, g]=\frac{\partial g}{\partial x} f-\frac{\partial f}{\partial x} g
$$

and define the involutive closure of a distribution $\Delta$ as the closure of $\Delta$ under Lie bracketing. Briefly, Chow's theorem states that if the involutive closure of the distribution associated with equation (1) spans $\mathbf{R}^{n}$ at each configuration, the system can be steered between any two configurations. Initial work in constructing paths between configurations includes $[8,10],[11]$, and $[9,17]$, as well as $[12,13]$. In this paper we concentrate on a different problem: stabilization to a point.

A control law $u=k(x, t)$ stabilizes a point $x_{0} \in \mathbf{R}^{n}$ if $x(t)-x_{0}$ as $t \rightarrow \infty$ for all initial conditions of the system. For a nonholonomic control system, the dependence of a stabilizing control law on time is essential since the system (1) does not satisfy Brockett's necessary condition for smooth stabilization [1]. Hence there does not exist a smooth static state feedback law which stabilizes the systen to a point. Recent work by Coron has shown that it is possible to stabilize a nonholonomic system using time-varying feedback [3]. Constructive approaches have

- Research supported in part by the Army under grant ARO DAAL-91-G-0191, and NASA under grant NAG2-243.

CH3229-2/92/0000-1603\$1.00 (C) 1992 IEEE been presented by Samson [15] and Pomet [14]. In this paper we present some new control laws for a specific class of systems, namely those in so-called chained form [13]. These control laws are based on earlier work using sinusoids for open-loop planning and have connections with the recent work in $[17]$

Chained systems. We restrict attention to a special class of nonholonomic systems, called chained systems [13]. A two-input system with a single chain has the form:

$$
\begin{aligned}
\dot{\xi}_{1} & =v_{1} \\
\dot{\xi}_{2} & =v_{2} \\
\dot{\xi}_{3} & =\xi_{2} v_{1} \\
\dot{\xi}_{4} & =\xi_{3} v_{1} \\
& \vdots \\
\dot{\xi}_{5} & =\xi_{n-1} v_{1} .
\end{aligned}
$$

This system is controllable using the input vector fields and Lie brackets of the form $\operatorname{ad}_{g_{1}}^{k} g_{2}$, where $\operatorname{ad}_{f} g$ is the iterated Lie bracket $[f,[f, \ldots,[f, g] \ldots]],(k$ copies of $f)$.

Under some conditions, it is possible to convert a two-input nonholonomic system into a system with the form of equation (2) using feedback transformations. Sufficient conditions for doing this are presented in [13]. In particular, it can be shown that under certain reg ularity conditions all two-input nonholonomic systems in $\mathbf{R}^{3}$ can be put into this form. More complicated examples of nonholonomic systems which are locally feedback equivalent to a chained form include kinematic models of an automobile and an automobile towing a trailer.

Chained systems can be steered between two arbitrary configurations using the following algorithm.

\section{Algorithm 1}

1. Steer $\xi_{1}$ and $\xi_{2}$ to their desired values.

2. For each $\xi_{k+2}, k \geq 1$, steer $\xi_{k+2}$ to its final value using $v_{1}=a \sin t$, $v_{2}=b \cos k t$, where $a$ and $b$ satisfy

$$
\xi_{k+2}(2 \pi)-\xi_{k+2}(0)=\frac{(a / 2)^{k} b}{k !} \cdot 2 \pi
$$

This algorithm uses $n$ path segments to steer the system. It is also possible to steer the system using a linear combination of sinusoidal terms at different frequencies by solving a polynomial equation for the coefficients of the sinusoids.

Power form. Related to chained form is a second canonical form which we refer to as "power form":

$$
\begin{aligned}
\dot{x}_{1} & =u_{1} \\
\dot{x}_{2} & =u_{2} \\
\dot{x}_{3} & =x_{1} u_{2} \\
\dot{x}_{4} & =\frac{1}{2} x_{1}^{2} u_{2} \\
& \vdots \\
\dot{x}_{n} & =\frac{1}{(n-2) !} x_{1}^{n-2} u_{2} .
\end{aligned}
$$

Like chained form, the control Lie algebra for this system is spanned by the input vector fields and Lie products of the form $\mathrm{ad}_{g_{1}}^{k} g_{2}$. The power form is related to the chained form through a global coordinate 
transformation:

$$
\begin{aligned}
x_{1} & =\xi_{1} \\
x_{2} & =\xi_{2} \\
x_{3} & =-\xi_{3}+\xi_{1} \xi_{2} \\
x_{4} & =\xi_{4}-\xi_{1} \xi_{3}+\frac{1}{2} \xi_{1}^{2} \xi_{2} \\
& \vdots \\
x_{n} & =(-1)^{n} \xi_{n}+\sum_{i=2}^{n-1}(-1)^{i} \frac{1}{(n-i) !} \xi_{1}^{n-i} \xi_{i}
\end{aligned}
$$

The advantage of using power form over chained form is that given $u_{1}$ and $u_{2}$, we can quickly solve for the motion of any of the state variables using only the trajectory of $x_{1}$ and the function $u_{2}$. This canonical form also arises in the work of Grayson and Grossman in the context of generating systems of vector fields which realize a nilpotent control Lie algebra of a given order [4]. It is also worthwhile to note that this form satisfies some of the simplifying assumptions used by Pomet to generate controllers for more general nonholonomic control systems [14].

In the sequel, we will restrict our results to those that apply to systems in chained form or, equivalently, power form. The are several reasons for taking this action. Systems which are in chained form characterize the fundamental difficulties of nonholonomic systems in a very simple and useful form. By understanding the geometry of controllers applied to chained form, we hope to understand the geometry of controllers applied to more general nonholonomic systems. This point of view has been used very successfully by Sussmann, who has shown how results applied to a "symbolic" representation of the control system can be used to understand systems with a compatible control Lie algebra [9]. Chained systems can be regarded as a realization of a class of "symbolic" control systems with a particular Lie algebraic structure.

The goal of this paper is to present a class of control laws with strong geometric intuition which asymptotically stabilize an arbitrary chained system with two inputs and a single chain. We are optimistic that the stabilizing controllers presented here can be extended to the more general case and that by understanding their action on a canonical system we can understand their extension to systems with a similar Lie algebraic structure.

\section{Local Stabilization}

In this section we propose a class of locally stabilizing inputs for (3). To motivate our approach, we consider first the simplest such system:

$$
\begin{aligned}
& \dot{x}_{1}=u_{1} \\
& \dot{x}_{2}=u_{2} \\
& \dot{x}_{3}=x_{1} u_{2}
\end{aligned}
$$

From the discussion of chained systems above, we know that motion in the $x_{3}$ direction can be achieved using sinusoidal inputs $u_{1}=a \sin t$ and $u_{2}=b \cos t$. Integrating the differential equations over one period, the resulting motion is a closed curve in $x_{1}$ and $x_{2}$ and a net motion of $-(a b) \pi$ in $x_{3}$. This suggests that the following control law

$$
\begin{aligned}
& u_{1}=-x_{1}-x_{3}^{2} \sin t \\
& u_{2}=-x_{2}-x_{3} \cos t
\end{aligned}
$$

might be used to stabilize the system. The intuition is that if $x_{3}$ is slowly varying then the average motion (over one period) in the $x_{3}$ coordinate can be approximated by setting $a=-x_{3}^{2}, b=-x_{3}$ which would give a net motion in $x_{3}$ of $-x_{3}^{3} \pi$, i.e., $x_{3}$ would converge to zero.

To prove stability in a more rigorous fashion we make use of center manifold theory and averaging. For the purposes of the proof, we realize the time-varying feedback law by augmenting the controller with an exosystem

and write the control law as

$$
\begin{array}{ll}
\dot{w}_{1}=w_{2} & w_{1}(0)=0 \\
\dot{w}_{2}=-w_{1} & w_{2}(0)=1,
\end{array}
$$

$$
\begin{aligned}
& u_{1}=-x_{1}-x_{3}^{2} w_{1} \\
& u_{2}=-x_{2}-x_{3} w_{2} .
\end{aligned}
$$

The closed loop system (including exosystem) has a local center manifold given by

$$
\begin{aligned}
& x_{1}=\pi_{1}\left(x_{3}, w_{1}, w_{2}\right) \\
& x_{2}=\pi_{2}\left(x_{3}, w_{1}, w_{2}\right),
\end{aligned}
$$

which is approximately given by

$$
\begin{aligned}
& \pi_{1}=-\frac{1}{2} x_{3}^{2}\left(w_{1}-w_{2}\right) \\
& \pi_{2}=-\frac{1}{2} x_{3}\left(w_{1}+w_{2}\right)
\end{aligned}
$$

The dynamics of the system evaluated on the center manifold are (approximately) given by

$$
\dot{x}_{3}=-\frac{1}{4} x_{3}^{3}\left(w_{1}-w_{2}\right)^{2} .
$$

An averaging-like coordinate change can then be made to show that the complete system is locally, asymptotically stable to the origin. For $x_{3}$ small, the higher order nature of $x_{3}^{3}$ plays the role of the small parameter $\epsilon$ usually found in averaging results.

We now consider the stabilization of an arbitrary system in power form. We begin with a local result and extend the controller to provide global convergence in the next section.

Theorem 2.1 Every pair of inputs

$$
\begin{aligned}
& u_{1}=-x_{1}-\left(\sum_{j=1}^{n-2} x_{j+2}^{2}\right)(\sin (t)-\cos (t)) \\
& u_{2}=-x_{2}-\sum_{j=1}^{n-2} c_{j} x_{j+2} \cos (j t)
\end{aligned}
$$

with $c_{j}>0$ locally asymptotically stabilizes the origin of (3).

Remark. The control law given in theorem 2.1 is a generalization of the simple controller presented earlier. We have added a cosine term to $u_{1}$ to make the proof tractable. It can be seen that, for the simple example, this extra term adds a term on the manifold of zero average. Sinusoids at integrally related frequencies are used to generate motion in the different bracket directions in such a way as to stabilize the system to the origin. We note that the control law requires neither the use of high-frequency sinusoids, such as those used by Sussmann and Liu for open loop steering [17] (see also [18]), nor does it require the use of a leading $\epsilon$ coefficient as typically used when applying averaging techniques. Likewise, compared to the work of [?], even though we employ an averaging like analysis, we do not require high-frequency sinusoids and we do not settle for stabilization to an arbitrarily small set. Furthermore, the weights $c_{j}$ can be adjusted to control the rate of convergence in the different coordinate directions in a straightforward manner.

Proof of theorem 2.1. The proof of theorem 2.1 will require applications of center manifold theory (see [2]), techniques used in averaging theory (see [5] or [7]) and a case specific Lyapunov result. Center manifold theory does not apply directly to $(3),(7)$ because the time-varying terms in (7) are $O(1)$. Nevertheless, we can demonstrate the following lemma regarding a class of systems to which (3), (7) can be transformed. We use the notation of [2] so that $f^{\prime}(0,0, w)$ refers to the partial derivative of $f$ with respect to all variables and evaluated at $(y, z, w)=(0,0, w)$.

Lemma 2.1 ("Time-varying" Center Manifold) Consider the system

$$
\begin{aligned}
\dot{y} & =B y+g(y, z, w) \\
\dot{z} & =A z+f(y, z, w) \\
\dot{w} & =S w
\end{aligned}
$$

with $y \in \mathbf{R}^{n}, z \in \mathbf{R}^{m}, w \in \mathbf{R}^{p}$ and where the eigenvalues of $B$ have negative real part and the eigenvalues of $A$ and $S$ have zero real part. The functions $f, g$ and $h$ are $C^{2}$ with $f(0,0, w)=0, f^{\prime}(0,0, w)=0$, $g(0,0, w)=0$, and $g^{\prime}(0,0, w)=0$. Then, given $M>0$, there exists a center manifold for (8), $y=h(z, w)$ for $|w|<M,|z|<\delta(M)$, for some $\delta>0$ and dependent on $M$, where $h$ is $C^{2}$ and $h(0, w)=0$, $h^{\prime}(0, w)=0$.

Proof. See appendix.

To transform (3), (7) into a system for which lemma 2.1 applies, we begin by defining $n-2$ linear oscillators which will generate the time-varying terms of (7). Let

$$
\dot{w}_{j}=\left[\begin{array}{c}
\dot{w}_{1 j} \\
\dot{w}_{2 j}
\end{array}\right]=\left[\begin{array}{cc}
0 & j \\
-j & 0
\end{array}\right]\left[\begin{array}{l}
w_{1 j} \\
w_{2 j}
\end{array}\right]=S_{j j} w_{j}
$$

We choose $w_{1 j}(0)=0, w_{2 j}(0)=1$ so that $w_{1 j}=\sin (j t)$ and $w_{2 j}=$ $\cos j t$. If we define the vector

$$
w=\left[\begin{array}{lll}
w_{1} & \ldots & w_{n-2}
\end{array}\right]^{T}
$$


we have

$$
\dot{w}=S w
$$

where $S$ is a block diagonal matrix with the $j$ th block given by $S_{j j}$. Next, partition the original state space as

$$
x=\left[\begin{array}{c}
x_{1} \\
x_{2} \\
--- \\
x_{3} \\
\vdots \\
x_{n}
\end{array}\right]=\left[\begin{array}{c}
y_{1} \\
y_{2} \\
--- \\
z
\end{array}\right]
$$

so that $y \in \mathbf{R}^{2}$ and $z \in \mathbf{R}^{m}$ with $m \equiv n-2$. For the closed loop system we have

$$
\begin{aligned}
\dot{y}_{1} & =-y_{1}-w^{T} D z^{T} z \\
\dot{y}_{2} & =-y_{2}-w^{T} C z \\
\dot{z} & =f(y, z, w) \\
\dot{w} & =S w
\end{aligned}
$$

where $f$ is $C^{2}$ with $f(0,0, w)=0$ and $f^{\prime}(0,0, w)=0$. The matrix $C \in \mathbf{R}^{2 m \times m}$ is block diagonal with the $j$ th block given by the column vector

$$
C_{j j}=\left[\begin{array}{c}
0 \\
c_{i}
\end{array}\right]
$$

and $D \in \mathbf{R}^{2 m}$ is given by

$$
D=\left[\begin{array}{lllll}
1 & -1 & 0 & \cdots & 0
\end{array}\right]^{T}
$$

We then make a coordinate change in $y_{2}$ to eliminate the linear timevarying dependence of $z$ in the $\dot{y}_{2}$ equation. We choose $\tilde{y}_{2}=y_{2}-z^{T} \mathrm{II}_{2} w$ where $\Pi_{2}$ solves the matrix equation

$$
\mathrm{I}_{2} S=-\mathrm{II}_{2} \quad C^{T}
$$

(The solution to this matrix equation always exists because the spectrum of $S$ is disjoint from the spectrum of $I$.) We then have

$$
\begin{aligned}
\dot{\tilde{y}}_{2} & =\dot{y}_{2}-z^{T} \Pi_{2} \dot{w}-\dot{z}^{T} \Pi_{2} w \\
& =-y_{2}-z^{T} C^{T} w-z^{T} \Pi_{2} S w-f^{T}(y, z, w) \Pi_{2} w \\
& =-y_{2}+z^{T} \Pi_{2} w-f^{T}(y, z, w) \Pi_{2} w \\
& =-\bar{y}_{2}+g_{2}(\bar{y}, z, w)
\end{aligned}
$$

where $g_{2}(0,0, w)=0$ and $g_{2}^{\prime}(0,0, w)=0$. We make the same kind of coordinate change for $y_{1}$. We choose $\bar{y}_{1}=y_{1}-z^{T} z \Pi_{1} w$ where $\mathrm{II}_{1}$ solves the matrix equation

$$
\Pi_{1} S=-I \Pi_{1}-D^{T}
$$

We then have

$$
\begin{aligned}
\dot{\tilde{y}}_{1} & =\dot{y}_{1}-z^{T} z \Pi_{1} \dot{w}-2 z^{T} \dot{z} \Pi_{1} w \\
& =-y_{1}-z^{T} z D^{T} w-z^{T} z \Pi_{1} s w-2 z^{T} f(y, z, w) \Pi_{1} w \\
& =-y_{1}+z^{T} z \Pi_{1} w-2 z^{T} f(y, z, w) \Pi_{1} w \\
& =-\tilde{y}_{1}+g_{1}(\bar{y}, z, w)
\end{aligned}
$$

where $g_{1}(0,0, w)=0, g_{1}^{\prime}(0,0, w)=0$ and $g_{1}^{\prime \prime}(0,0, w)=0$.

Now, from lemma 2.1 there is a center manifold $\tilde{y}=h(z, w),|z|<$ $\delta,|w|<M$ for

$$
\begin{aligned}
\dot{\tilde{y}} & =-I \tilde{y}+g(\bar{y}, z, w) \\
\dot{z} & =\tilde{f}(\tilde{y}, z, w) \\
\dot{w} & =S w
\end{aligned}
$$

where $h(0, w)=0$ and $h^{\prime}(0, w)=0$. In fact, since $g_{1}^{\prime \prime}(0,0, w)=0$, one can use an approximation theorem [2, theorem 3$]$ or calculate to show that $h_{1}^{\prime \prime}(0, w)=0$ (where $h=\left[h_{1}, h_{2}\right]^{T}$ ). Now it is sufficient to analyze the dynamics of the reduced system

$$
\begin{aligned}
\dot{z} & =\bar{f}(h(z, w), z, w) \\
\dot{w} & =S w
\end{aligned}
$$

Further, since $h(0, w)=0$ and the dynamics of $w$ are autonomous with $|w(t)|<M$ for all $t \geq 0$ for some $M>0$, it is sufficient to check the stability of $z=0$ for the following "time-varying" nonlinear differential equation:

$$
\begin{aligned}
\dot{z}_{1} & =\left(h_{1}+z^{T} z \Pi_{1} w\right)\left(-h_{2}+w^{T} S^{T} \Pi_{2}^{T} z\right) \\
& \vdots \\
\dot{z}_{m} & =\frac{1}{m !}\left(h_{1}+z^{T} z \Pi_{1} w\right)^{m}\left(-h_{2}+w^{T} S^{T} \Pi_{2}^{T} z\right)
\end{aligned}
$$

First, becausc $h(0, w)=0, h^{\prime}(0, w)=0$ and $h_{1}^{\prime \prime}(0, w)=0$ we can write the dynamics of $z$ as

$$
\begin{aligned}
\dot{z}_{1} & =\left(z^{T} z \Pi_{1} w\right) z^{T} \Pi_{2} S w+O(z)^{4} \\
\dot{z}_{2} & =\frac{1}{2}\left(z^{T} z \Pi_{1} w\right)^{2}\left(z^{T} \Pi_{2} S w\right)+O(z)^{6} \\
& \vdots \\
\dot{z}_{m} & =\frac{1}{m !}\left(z^{T} z \Pi_{1} w\right)^{m}\left(z^{T} \Pi_{2} S w\right)+O(z)^{2(m+1)}
\end{aligned}
$$

Now we determine expressions for $\Pi_{1}$ and $\Pi_{2}$ to examine the explicit time dependence of $(22)$. From the block structure of $S$ and $C$ it follows that $\Pi_{2}$ also has a block structure where the $j$ th block satisfies the matrix equation

$$
\mathrm{II}_{2, j} S_{j j}=-I \mathrm{H}_{2 j}-C_{j j}^{T}
$$

It can be shown that

$$
\Pi_{2 j}=\left[-\frac{j}{1+j^{2}} c_{j}-\frac{1}{1+j^{2}} c_{j}\right]
$$

and, hence,

$$
\mathrm{II}_{2 j J} S_{j j}=\left[\frac{j}{1+j^{2}} c_{j}-\frac{j^{2}}{1+j^{2}} c_{j}\right]
$$

Thus we have

$$
w^{T} S^{T} \Pi_{2}^{T} z=\sum_{j=1}^{m} c_{j}\left[\frac{j}{1+j^{2}} \sin (j t)-\frac{j^{2}}{1+j^{2}} \cos (j t)\right] z_{j}
$$

Now from (17) it can be shown that

$$
\Pi_{1}=\left[\begin{array}{lllll}
0 & 1 & 0 & \cdots & 0
\end{array}\right]
$$

so that $z^{T} z \Pi_{1} w=z^{T} z \cos (t)$. We now consider the product

$$
\frac{1}{i !}\left(z^{T} z \Pi_{1} w\right)^{i}\left(w^{T} S^{T} \Pi_{2}^{T} z\right)
$$

given by

$$
\frac{1}{i !}\left(z^{T} z \cos (t)\right)^{2}\left(\sum_{j=1}^{m} c_{j}\left[\frac{j}{1+j^{2}} \sin (j t)-\frac{j^{2}}{1+j^{2}} \cos (j t)\right] z_{j}\right)
$$

Using the identity

$$
\cos (t) \cos (k t)=\frac{1}{2}[\cos ((k-1) t)+\cos ((k+1) t)]
$$

it can be shown that

$$
\cos ^{i}(t)=\sum_{k=1}^{\ell} \alpha_{i k} \cos ([i-2(k-1)] t)
$$

where $\alpha_{i k}>0$ and $\ell=\frac{t}{2}+1$ if $i$ is even and $\frac{i+1}{2}$ if $i$ is odd.

At this point, we would like to apply averaging to the terms in (24) to conclude asymptotic stability. However, since we are not using high frequency sinusoids and we do not have exponential stability for the averaged system, general averaging results do not apply. Nevertheless, a very specific averaging result which covers the class of systems we have can be asserted. We describe this result in the next two lemmas. The uniformly higher order characteristic of our equations eliminates the need for a small parameter (or alternatively, very high frequencies). We are able to find a case specific Lyapunov function that demonstrates asymptotic stability in the presence of small time-varying disturbances without requiring exponential stability.

Lemma 2.2 ("Averaging" transformation) Consider the timevarying nonlinear system

$$
\dot{x}=f(x, t)
$$

where $f$ is of period $T$ in $t$ and is $C^{r}$ and the ith entry of the vector $f$ satisfies $f_{i}=O(x)^{2 i+1}$. Then there exists a $C^{r}$ local change of coordinates $x=y+\Psi(y, t)$ under which (26) becomes

$$
\dot{y}=\bar{f}(y)+\hat{f}(y, t)
$$

where $\bar{f}$ is the time average of $f$ and $\hat{f}_{i}(y, t)=O(y)^{2 i+2}$ and of period $T$ in $t$.

Proof. See appendix. 
Lemma 2.3 (Case Specific Lyapunov result) Consider the system

$$
\dot{y}=\bar{f}(y)+\tilde{f}(y, t)
$$

where $y \in \mathbf{R}^{n}$. If

$$
\left|\tilde{f}_{i}(y, t)\right| \leq \beta_{i}\|y\|^{2(1+i)}
$$

for all $y$ in some open neighborhood of the origin and

$$
\tilde{f}(y)=A \psi(y)
$$

where $A$ is a square lower triangular matrix with $a_{i i}<0$ for $i=1, \ldots, n$ and

$$
\psi_{i}(y)=y_{i}|| y \|^{2 i}
$$

then the origin of (28) is locally asymptotically stable.

Proof. See appendix.

Now we make the coordinate transformation of lemma 2.2 to pull out the lowest order terms on each line of equation (22) with nonzero average. Using (24) and (25) we can show that this transformation yields a system possessing the (triangular) structure of the system in lemma 2.3. In fact, the $a_{i j}$ 's of lemma 2.3 are given by

$$
a_{j j}=-\frac{1}{2 j !} \frac{j^{2}}{1+j^{2}} \alpha_{j 1} c_{j}
$$

Since $\alpha_{j 1}, c_{j}>0$, the local asymptotic stability of the origin of (3), (7) then follows from lemma 2.3. $\square$

\section{Global Stabilization}

In this section we propose a class of smooth, time-varying, globally stabilizing inputs for (3). Near the origin these control laws will exactly match the locally stabilizing control laws proposed in section 2 . We introduce saturation functions in these control laws to eliminate destabilizing effects that take place away from the origin.

Theorem 3.1 Given any pair of inputs

$$
\begin{aligned}
& u_{1}=-x_{1}-\sigma\left(\left(\sum_{j=1}^{n-2} x_{j+2}^{2}\right)^{\frac{1}{2}}\right)^{2}(\sin (t)-\cos (t)) \\
& u_{2}=-x_{2}-\sum_{j=1}^{n-2} c_{j} \sigma\left(x_{j+2}\right) \cos (j t)
\end{aligned}
$$

with $c_{j}>0$ and with $\sigma: \mathbf{R} \rightarrow \mathbf{R}$ a nondecreasing $C^{3}$ function satisfying

1. $\sigma(s)=s$ when $|s| \leq \delta$

2. $|\sigma(s)| \leq \epsilon$ for all $s \in \mathbf{R}$

for some $0<\delta<\epsilon, \exists \epsilon_{0}$ such that if $\epsilon<\epsilon_{0}$ then the origin of (3) is globally asymptotically stable.

Proof of theorem 3.1. The proof of theorem 3.1 is very much in the spirit of the proof of theorem 2.1. We begin by defining the same oscillators as in (9) and we make the same partition of the state space as in (11). For (3), (32) we have

$$
\begin{aligned}
\dot{y}_{1} & =-y_{1}-w^{T} D \sigma(\|z\|)^{2} \\
\dot{y}_{2} & =-y_{2}-w^{T} C \bar{\sigma}(z) \\
\dot{z} & =f(y, z, w) \\
\dot{w} & =S w
\end{aligned}
$$

where

$$
\bar{\sigma}(z)=\left[\begin{array}{lll}
\sigma\left(z_{1}\right) & \cdots & \sigma\left(z_{m}\right)
\end{array}\right]^{T}
$$

The matrices $C$ and $D$ are as defined in (13) and (14) respectively.

We make the coordinate change

$$
\begin{aligned}
& \tilde{y}_{1}=y_{1}-\sigma(|| z||)^{2} \Pi_{1} w \\
& \tilde{y}_{2}=y_{2}-\bar{\sigma}^{T}(z) \Pi_{2} w
\end{aligned}
$$

where $\Pi_{1}$ and $\Pi_{2}$ satisfy (17) and (15) respectively.

We then have

$$
\begin{aligned}
\dot{y}_{1} & =-\tilde{y}_{1}-2 \sigma(\|z\|) \frac{\partial \sigma}{\partial\|z\|}\|z\|^{-1} z^{T} f(y, z, w) \Pi_{1} w \\
& =-\tilde{y}_{1}+g_{1}(\tilde{y}, z, w) \\
\dot{y}_{2} & =-\tilde{y}_{2}-f^{T}(y, z, w) \frac{\dot{\sigma}^{T}}{\partial z} \Pi_{2} w \\
& =-\tilde{y}_{2}+g_{2}(\tilde{y}, z, w)
\end{aligned}
$$

We now wish to show that given $\epsilon$ sufficiently small, there is a center manifold $\tilde{y}=h(z, w), z \in \mathbf{R}^{m},|w|<M$ for

$$
\begin{aligned}
\dot{\tilde{y}} & =-I \tilde{y}+g(\tilde{y}, z, w) \\
\dot{z} & =\tilde{f}(\tilde{y}, z, w) \\
\dot{w} & =S w
\end{aligned}
$$

where $h(0, w)=0$ and $h^{\prime}(0, w)=0$. To do so, following the proof of $[2$, theorem 1], we must show that given $M>0$ and for $\epsilon$ sufficiently small, there exists a continuous function $\kappa(\epsilon)$ with $\kappa(0)=0$ such that

$$
\begin{aligned}
|\tilde{f}(\tilde{y}, z, w)|+|g(\tilde{y}, z, w)| & \leq \epsilon \kappa(\epsilon) \\
\left|\tilde{f}(\tilde{y}, z, w)-\tilde{f}\left(\tilde{y}^{\prime}, z^{\prime}, w^{\prime}\right)\right| & \leq \kappa(\epsilon)\left(\left|\tilde{y}-\tilde{y}^{\prime}\right|+\left|z-z^{\prime}\right|+\left|w-w^{\prime}\right|\right) \\
\left|g(\tilde{y}, z, w)-g\left(\tilde{y}^{\prime}, z^{\prime}, w^{\prime}\right)\right| & \leq \kappa(\epsilon)\left(\left|\tilde{y}-\tilde{y}^{\prime}\right|+\left|z-z^{\prime}\right|+\left|w-w^{\prime}\right|\right)
\end{aligned}
$$

for all $z, z^{\prime} \in \mathbf{R}^{m}$, and all $w, w^{\prime} \in \mathbf{R}^{p}$ with $|w|,\left|w^{\prime}\right|<M$ and all $y, y^{\prime} \in$ $\mathbf{R}^{n}$ with $|y|,\left|y^{\prime}\right|<\epsilon$. It can be shown that $\tilde{f}$ satisfies this relationship, since every dependence on $z$ in $\tilde{f}$ is as the argument of a saturation function bounded by $\epsilon$. Then, since $\tilde{f}$ satisfies these relationships, it follows from (34) that $g$ also satisfies these relationships by noting that $\sigma$ is $C^{3}$ and hence its partials are bounded and $\left|\frac{z}{\|z\|}\right| \leq b$ for some positive constant $b$.

Next we show that, for $\epsilon$ sufficiently small, the manifold $h(z, w)$ is globally attractive. First, observe that the dynamics of $y$ are of an exponentially stable linear system perturbed by bounded disturbances of magnitude proportional to $\epsilon$. Consequently, after some finite time $y$ is contained in a ball of radius proportional to $\epsilon$. Then, by the nature of the coordinate change from $y$ to $\tilde{y}, \tilde{y}$ is also contained in a ball of radius proportional to $\epsilon$. Now we know that the manifold is locally attractive, so for $\epsilon$ sufficiently small the $\epsilon$ ball is contained in the basin of attraction for $h(z, w)$. Hence, the manifold $h(z, w)$ is globally attractive.

We will eventually establish that the dynamics

$$
\dot{z}=\tilde{f}(h(z, w)+(\tilde{y}-h(z, w)), z, w)
$$

have the "converging input bounded state" property of [16] with $e \equiv$ $\tilde{y}-h(z, w)$ as input. Then, since $h(0, w)=0$ it is sufficient to consider the dynamics of

$$
\dot{z}=\tilde{f}(h(z, w), z, w)
$$

For now, we simply consider the global stability property of (38). To do so, we begin by establishing a bound on $h(z, w)$. We follow the approximation of center manifolds in [2]. As in [2], for functions $\phi$ : $\mathbf{R}^{n} \times \mathbf{R}^{p} \rightarrow \mathbf{R}^{2}$ which are $C^{1}$ in a neighborhood of the origin we define the operator $N$ to be

$(N \phi)(z, w)=\frac{\partial \phi}{\partial z} \tilde{f}(\phi(z, w), z, w)+\frac{\partial \phi}{\partial w} S w+I \phi(z, w)-g(\phi(z, w), z, w)$ where $g$ is defined in (34). We choose to approximate $h(z, w)$ by the function $\phi(z, w) \equiv 0$. We then have

$$
(N \phi)(z, w)=-g(0, z, w)
$$

It follows from $(34)$ and $\tilde{f}$ that $(N \phi)(z, w)=O\left(\sigma(\|z\|)^{3}\right)$ for all $z \in \mathbf{R}^{m}$ and all $w \in \mathbf{R}^{p}$ with $|w|<M$. We can then mimic the proof of $[2$, theorem 3] to establish that

$$
|h(z, w)-\phi(z, w)|=|h(z, w)|=O\left(\sigma(\|z\|)^{3}\right)
$$

for all $z \in \mathbf{R}^{m}$ and all $w \in \mathbf{R}^{p}$ with $|w|<M$.

We are now ready to establish lemmas similar to lemmas 2.1 and 2.2 that apply to the global stability problem.

Lemma 3.1 (Global “Averaging" transformation) Consider the nonlinear time-varying system

$$
\dot{x}=f(x, t)
$$

where $f$ is of period $T$ in $t$ and is $C^{r}$ and where the ith entry of the vector $f$ satisfies $f_{i}=O\left(\sigma(\|x\|)^{2 i+1}\right)$. If the $\epsilon$ associated with the saturation function $\sigma$ is sufficiently small, then there exists a $C^{\tau}$ global change of coordinates $x=y+\Psi(y, t)$ under which (40) becomes

$$
\dot{y}=\bar{f}(y)+\hat{f}(y, t)
$$

where $\vec{f}$ is the time average of $f$ and $\hat{f}$ is of period $T$ in $t$ with $\hat{f}_{i}(y, t)=$ $O\left(\sigma(\|y\|)^{2 i+2}\right)$. 
Proof. See appendix.

Lemma 3.2 (Global Case Specific Lyapunov result) Consider the system

$$
\dot{y}=\bar{f}(y)+\tilde{f}(y, t)
$$

where $y \in \mathbf{R}^{n}$. If

$$
\left|\tilde{f}_{i}(y, t)\right| \leq \beta_{i} \sigma\left(\|y\|_{1}\right)^{2(1+i)}
$$

for all $y \in \mathbf{R}^{n}$ and

$$
f(y)=A \psi(y)
$$

where $A$ is a square lower triangular matrix with $a_{i i}<0$ for $i=1, \ldots, n$ and

$$
\psi_{i}(y)=\sigma\left(y_{i}\right) \sigma(|| y||)^{2 i}
$$

then, for $\epsilon$ sufficiently small, the origin of (42) is globally asymptotically stable.

Proof. See appendix.

Now using the expression for $\Pi_{1}$ and $\Pi_{2}$ from the proof of theorem 2.1 we can show that these lemmas apply and thus the reduced dynamics are globally asymptotically stable. It remains to verify that the $z$ dynamics have the "converging input bounded state" property of [16]. Since $\bar{f}$ is bounded for bounded $\epsilon$, and hence $z$ is bounded for all finite time, it is sufficient to prove the following result:

Lemma 3.3 (Converging input bounded state) Under the conditions of lemma 3.2 , if the perturbation in the equation

$$
\dot{y}=\bar{f}(y)+\tilde{f}(y, t)+p(t)
$$

satisfies $|p(t)| \leq \nu$, then, for $\nu$ sufficiently small, $y$ satisfies $|y(t)| \leq G$ for all $t \geq 0$ for some $G>0$.

Proof. See appendix.

Now the main theorem of [16] provides global asymptotic stability for the system (3), (32). $\square$

It is also possible to deduce a locally stabilizing control law for (2) without using the transformation to power form given in (4).

Corollary 3.1 Every pair of inputs

$$
\begin{aligned}
& v_{1}=-\xi_{1}-\left(\sum_{j+1}^{n-2} \xi_{j+2}^{2}\right)(\sin (t)-\cos (t)) \\
& v_{2}=-\xi_{2}-\sum_{j=1}^{n-2}(-1)^{j} c_{j} \xi_{j+2} \cos (j t)
\end{aligned}
$$

with $c_{j}>0$ locally asymptotically stabilizes the origin of (2).

Proof of corollary 3.1. Let the transformation (4) that takes us from chain form to power form be written as $x=\Phi(\xi)=T \xi+\bar{\Phi}(\xi)$ where $\bar{\Phi}(\xi)$ is higher order. Let $v_{\text {chain }}(\cdot)$ denote the controls given by (47) and let $u_{\text {power }}(\cdot)$ denote the controls given by 7 . Then we have $v_{\text {chain }}(\xi)=u_{\text {power }}\left(T^{-1} x\right)$. For (2). (47) if we make the transformation $x=\Phi(\xi)$, we have a power form system (3) with controls given by (7) plus higher order terms. Now the proof is exactly equivalent to the proof of theorem 2.1 since the higher order terms would simply contribute higher order terms on the manifold which were shown to be unimportant. $\square$

\section{Example: an automobile}

Our example system will be a simple kinematic model of an automobile as shown in figure 1. This system is controllable using two levels of Lie Brackets. A derivation of the kinematic equations may be found in [12]. A sketch of the car is found in Figure 1

$$
\begin{aligned}
\dot{x} & =\cos (\theta) u_{1} \\
\dot{y} & =\sin (\theta) u_{1} \\
\dot{\phi} & =u_{2} \\
\dot{\theta} & =\frac{1}{L} \tan (\phi) u_{1}
\end{aligned}
$$

where $(x, y)$ is the position of the car in the plane, $\phi$ is the angle of the front wheels with respect to the car (or the steering wheel angle), $\theta$ is the orientation of the car with respect to some reference frame, and the constant $L$ is the length of the wheel base. For simplicity, we choose $L=1$.

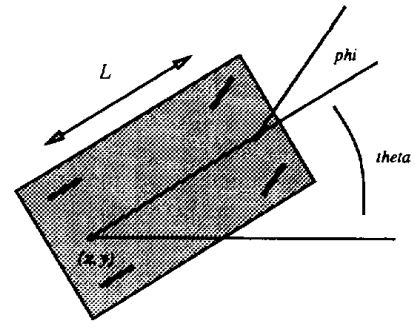

Figure 1: Kinematic model of the car

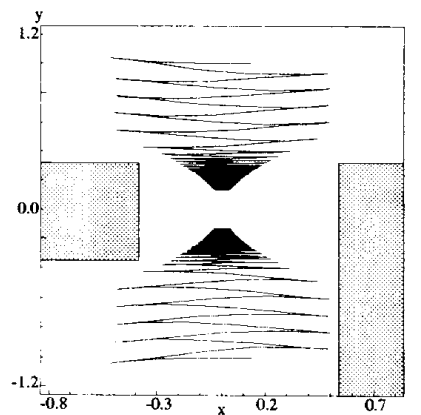

Figure 2: Phase plane plot, $x$ versus $y$, of the two simulations. Note the effects of the saturation function on the limits of travel in the $x$ direction.

The following change of coordinates will put the car into power form coordinates, locally:

$$
\begin{aligned}
& x_{1}=x \\
& x_{2}=\sec ^{3}(\theta) \tan (\phi) \\
& x_{3}=x \sec ^{3}(\theta) \tan (\phi)-\tan (\theta) \\
& x_{4}=y+\frac{1}{2} x^{2} \sec ^{3}(\theta) \tan (\phi)-x \tan (\theta)
\end{aligned}
$$

with the following input transformation:

$$
\begin{aligned}
& u_{1}=v_{1} \sec (\theta) \\
& u_{2}=-3 v_{1} \sec (\theta) \sin ^{2}(\phi) \tan (\theta)+v_{2} \cos ^{3}(\theta) \cos ^{2}(\phi)
\end{aligned}
$$

The control law used for the simulation was:

$$
\begin{aligned}
& v_{1}=-x_{1}-\sigma^{2}\left(\sqrt{x_{3}^{2}+x_{4}^{2}}\right)(\sin (t)-\cos (t)) \\
& v_{2}=-x_{2}-k \sigma\left(x_{3}\right) \cos (t)-k \sigma\left(x_{4}\right) \cos (2 t)
\end{aligned}
$$

The gain $k$ was chosen to be 2 , and the $\epsilon$ of the saturating function $\sigma(\cdot)$ was chosen to be $\epsilon=0.5$. The initial conditions chosen for these two simulations were $(0, \pm 1,0.0)$. The plot demonstrates the effect using a saturation function. At first the error is large enough to cause the saturation functions to limit the magnitude of the input sinusoids, hence limiting the $x$ and $\phi$ travel of of the car. After the error drops sufficiently, the controls are no longer saturated and the range of travel drops.

\section{Summary and Discussion}

We have presented a control law which globally asymptotically stabilizes a system in power form. This control law uses sinusoids at integrally related frequencies to achieve motion in bracketing directions and saturation functions to achieve globally convergence. Convergence in the coordinate directions can be adjusted by setting the appropriate weights in the control law. By making use of a feedback transformation to convert a nonholonomic system into power form, we have applied this control law to a kinematic model of an automobile.

The primary limitation of the control law presented here is that it can only be applied to systems which are feedback equivalent to a 
system in power form. However, there is strong evidence to suggest that control laws of this form can be extended to more general nonholonomic systems by using an "extended system" such as that used by Sussmann and co-workers $[9,17]$. The generalization of the results presented here would be to systems which are controllable through the input vector fields and Lie products of the form $\operatorname{ad}_{g_{1}}^{k} g_{2}$. Controllers for this same basic class of systems can be found in the recent work of Pomet [14] The extension of the ideas presented here to this more general situation is the subject of current research.

\section{Appendix}

\subsection{Proof of lemma 2.1}

The proof of lemma 2.1 mimics the proof of [2, Theorem 1, pp. 16-19] Accordingly, let $\psi: \mathbf{R}^{+} \rightarrow[0,1]$ be a $C^{\infty}$ function with $\psi(s)=1$ when $s \leq 1$ and $\psi(s)=0$ when $s \geq 2$. Then for $\epsilon, M>0$ define $F$ and $G$ by

$$
\begin{aligned}
& F(y, z, w)=f\left(y, z \psi\left(\frac{|z|}{\epsilon}\right), w \psi\left(\frac{|w|}{M}\right)\right) \\
& G(y, z, w)=g\left(y, z \psi\left(\frac{|z|}{\epsilon}\right), w \psi\left(\frac{|w|}{M}\right)\right)
\end{aligned}
$$

We prove that, given $M>0$, the system

$$
\begin{aligned}
\dot{y} & =B y+G(y, z, w) \\
\dot{z} & =A z+F(y, z, w) \\
\dot{w} & =S w
\end{aligned}
$$

has a center manifold $y=h(z, w), z \in \mathbf{R}^{m}, w \in \mathbf{R}^{p}$ for $\epsilon$ sufficiently small. Then since $F$ and $G$ agree with $f$ and $g$ for all $|z|<\epsilon$ and for all $|w|<M$, this proves the existence of a local center manifold for (8). The existence of the global center manifold for (49) can be demonstrated using the same contraction mapping calculations as in the proof of [2, theorem 1] since we can show, as was needed in [2] that there is a continuous function $\kappa(\epsilon)$ with $\kappa(0)=0$ such that

$$
\begin{aligned}
& |F(y, z, w)|+|G(y, z, w)| \leq \epsilon(\epsilon) \\
& \mid F(y, z, w)-F\left(y^{\prime}, z^{\prime}, w^{\prime} \mid \leq \kappa(\epsilon)\left(\left|y-y^{\prime}\right|+\left|z-z^{\prime}\right|+\left|w-w^{\prime}\right|\right)\right. \\
& \mid G(y, z, w)-G\left(y^{\prime}, z^{\prime}, w^{\prime} \mid \leq \kappa(\epsilon)\left(\left|y-y^{\prime}\right|+\left|z-z^{\prime}\right|+\left|w-w^{\prime}\right|\right)\right.
\end{aligned}
$$

for all $z, z^{\prime} \in \mathbf{R}^{m}$, and all $w, w^{\prime} \in \mathbf{R}^{p}$ and all $y, y^{\prime} \in \mathbf{R}^{n}$ with $|y|,\left|y^{\prime}\right|<\epsilon$ Following [2], these inequalities yield a center manifold $y=h(z, w)$ with $h(0, w)=0$ and $h^{\prime}(0, w)=0$.

\subsection{Proof of lemma 2.2}

The proof of this lemma follows closely the exposition of [5, pp. 168 169]. We split $f(x, t)$ as

$$
f(x, t)=\bar{f}(x)+\tilde{f}(x, t)
$$

where $\bar{f}$ is the mean of $f$ and $\tilde{f}$ is its oscillating part. Now we make the coordinate change

$$
x=y+\Psi(y, t)
$$

where $\Psi$ will be specified. (We will show $\Psi$ to be strictly higher order so that this is a valid coordinate change locally.) Differentiating we have

$$
\left[I+D_{y} \Psi\right] \dot{y}+\frac{\partial \Psi}{\partial t}=\dot{x}=\bar{f}(y+\dot{\Psi})+\tilde{f}(y+\Psi, t)
$$

Reorganizing we get

$$
\left.\dot{y}=\left[I+D_{y} \Psi\right]^{-1}\right]\left[\bar{f}(y+\Psi)+\tilde{f}(y+\Psi, t)-\frac{\partial \Psi}{\partial t}\right]
$$

We now choose $\Psi$ such that

$$
\frac{\partial \Psi}{\partial t}=\tilde{f}(y, t)
$$

(Since $\tilde{f}$ has zero mean, $\Psi$ is bounded as a function of time.) This choice produces

$$
\dot{y}=\left[I+D_{y} \Psi\right]^{-1}[\bar{f}(y)+f(y+\Psi, t)-f(y, t)]
$$

Expanding with respect to $\Psi$ we have

$$
\begin{aligned}
\dot{y} & =\left[I-D_{y} \Psi+O\left(\left\|D_{y} \Psi\right\|^{2}\right)\right]\left[\bar{f}+D_{y} f \Psi+O\left(\|\Psi\|^{2}\right)\right] \\
& \equiv \bar{f}(y)+\hat{f}(y, t)
\end{aligned}
$$

Now we check the order of $\hat{f}_{i}$. The first term we consider is the term

$$
I[f(y+\Psi, t)-f(y, t)]
$$

It suffices to check the order of the $i$ th entry of

$$
D_{y} f \cdot \Psi
$$

Accordingly, the entries of the $i$ th row of $D_{y} f$ are of order $2 i$. Further, since

$$
\frac{\partial \Psi_{i}}{\partial t}=\bar{f}_{i}(y, t)
$$

it follows that $\Psi_{i}$ is of order $1+2 i$ in $y$. Hence, the lowest order in $w$ is $3(i=1)$ and the product yields terms of order $2 i+3$.

The final terms we need to consider are given by $D_{y} \Psi N(y, t)$ where

$$
N_{i}(y, t) \equiv \bar{f}_{i}(y)+f_{i}(y+\Psi, t)-f_{i}(y, t)
$$

By assumption, we know that $N_{i}(y, t)$ is of order $1+2 i$ in $y$. Since $\Psi_{i}$ is of order $1+2 i$ it follows that the entries of the $i$ th row of $D_{y} \Psi$ are of order $2 i$. The lowest order in $N(y, t)$ is $3(i=1)$ and so the $i$ th entry of $D_{y} \Psi N(y, t)$ is of order $2 i+3$.

\subsection{Proof of lemma 2.3}

Consider the Lyapunov function

$$
V=\sum_{i=1}^{n} \frac{\alpha_{i}}{2(n+1-i)} y_{i}^{2(n+1-i)}
$$

where the $\alpha_{i}$ 's will be specified later. The derivative of $V$ along the trajectories of $(28)$ is given by

$$
\begin{aligned}
\dot{V} & =\sum_{i=1}^{n} \alpha_{i} y_{i}^{2(n-i)+1}\left[\bar{f}_{i}(y)+\tilde{f}_{i}(y, t)\right] \\
& \leq\left[\sum_{i=1}^{n} \alpha_{i} y_{i}^{2(n-i)+1} A_{i} \psi(y)\right]+\gamma\|y\|^{2(n+1)+1}
\end{aligned}
$$

where $A_{i}$ is the $i$ th row of the matrix $A$ and $\gamma$ is a constant that depends on $\alpha_{i}, \beta_{i}$ for $i=1, \ldots, n$. We claim that the $\alpha_{i}$ 's can be chosen such that

$$
\mathcal{S}(y) \equiv \sum_{i=1}^{n} \alpha_{i} y_{i}^{2(n-i)+1} A_{i} \psi(y) \leq-\|y\|^{2(n+1)}
$$

This will give

$$
\dot{V} \leq-(1-\gamma\|y\|)\|y\|^{2(n+1)}
$$

and hence asymptotic stability of the origin for $\|y\|$ sufficiently small. The proof of this claim will involve an iterative process of completing squares, bookkeeping coefficients and judiciously choosing the $\alpha_{i}$ 's.

We begin by multiplying the $i$ th term $(i=1, \ldots, n)$ in the summation $\mathcal{S}(y)$ by

for $\|y\| \neq 0$. This yields

$$
\left(\frac{\|y\|}{\|y\|}\right)^{2(n-i+1)}
$$

$$
\mathcal{S}(y)=\|y\|^{2(n+1)} \sum_{i=1}^{n}\left[\alpha_{i} \frac{y_{i}^{2(n-i)+1}}{\|y\|^{2(n-i+1)}} \sum_{j=1}^{i} a_{i j} y_{j}\right] \equiv\|y\|^{2(n+1)} \mathcal{T}(y)
$$

Now we begin to complete squares by first considering the quadratic terms (i.e. those terms generated by $i=n$ in the summation). Doing so, we have

$$
\begin{aligned}
\mathcal{T}(y) \leq & {\left[\sum_{i=1}^{n-1} \alpha_{i} \frac{y_{i}^{2(n-i)+1}}{\|y\|^{2(n-i+1)}} \sum_{j=1}^{i} a_{i j} y_{j}\right] } \\
& +\alpha_{n} \frac{a_{n n}}{2}\left(\frac{y_{n}}{\|y\|}\right)^{2}+\alpha_{n} \sum_{j=1}^{n-1} \tilde{a}_{n j}\left(\frac{y_{j}}{\|y\|}\right)^{2}
\end{aligned}
$$

Here $\tilde{a}_{n j}$ are positive constants that depend on $a_{n n}, a_{n j}$, ahd $n$. Now, by the definition of $\|y\|$, we have

$$
y_{n}^{2}=\|y\|^{2}-y_{1}^{2}-\ldots-y_{n-1}^{2}
$$

and choosing

$$
\alpha_{n}=-\frac{4}{a_{n n}}
$$

we have

$$
\mathcal{T}(y) \leq\left[\sum_{i=1}^{n-1} \alpha_{i} \frac{y_{i}^{2(n-i)+1}}{\|y\|^{2(n-i+1)}} \sum_{j=1}^{i} a_{i j} y_{j}\right]-2+\alpha_{n} \sum_{j=1}^{n-1} \tilde{a}_{n j}\left(\frac{y_{j}}{\|y\|}\right)^{2}
$$


with the $\tilde{\boldsymbol{a}}_{n j}$ 's appropriately redefined positive constants.

Now we consider the quartic terms generated by $i=n-1$ in the summation. Again completing squares, and using the fact that

$$
\left(\frac{y_{n-1}}{\|y\|}\right)^{2}\left(\frac{y_{k}}{\|y\|}\right)^{2} \leq\left(\frac{y_{k}}{\|y\|}\right)^{2}
$$

we have

$$
\begin{aligned}
\mathcal{T}(y) \leq & -2+\left[\sum_{i=1}^{n-2} \alpha_{i} \frac{y_{i}^{2(n-i)+1}}{\|y\|^{2(n-i+1)}} \sum_{j=1}^{i} a_{i j} y_{j}\right]+\alpha_{n}\left[\sum_{j=1}^{n-1} \tilde{a}_{n j}\left(\frac{y_{j}}{\|y\|}\right)^{2}\right] \\
& +\alpha_{n-1} \frac{a_{n-1, n-1}}{2}\left(\frac{y_{n-1}}{\|y\|}\right)^{4}+\alpha_{n-1} \sum_{j=1}^{n-2} \tilde{a}_{n-1, j}\left(\frac{y_{j}}{\|y\|}\right)^{2}
\end{aligned}
$$

We now choose $\alpha_{n-1}$ sufficiently large so that

$$
\alpha_{n-1} \frac{a_{n-1, n-1}}{2}\left(\frac{y_{n-1}}{\|y\|}\right)^{4}+\alpha_{n} \tilde{a}_{n, n-1}\left(\frac{y_{n-1}}{\|y\|}\right)^{2} \leq \frac{1}{n-1}
$$

In fact, we continue this process of completing squares and choosing $\alpha_{i}$ large enough such that all the terms involving $y_{i}$ are bounded by $\frac{1}{n-1}$. This can always be done because of the triangular structure. Finally we have that

$$
\mathcal{T}(y) \leq-2+\sum_{i=1}^{n-1} \frac{1}{n-1} \leq-1
$$

From this we conclude that

$$
S(y) \leq-\|y\|^{2(n+1)}
$$

for $\|y\| \neq 0$ and our claim is established. $\square$

\subsection{Proof of lemma 3.1}

The proof of this lemma is a virtual duplication of the proof of lemma 2.2. We split $f$ as before and make a similar coordinate change

$$
x=y+\Psi(y, t)
$$

This time we will establish that for $\epsilon$ sufficiently small, this is a globally valid coordinate transformation. In fact, we again pick

$$
\frac{\partial \Psi}{\partial t}=\tilde{f}(y, t)
$$

Since $\tilde{f}_{i}(y, t)=O\left(\sigma(\|y\|)^{2 i+1}\right)$ and $\sigma$ is $C^{3}$ it follows that $\Psi=$ $O\left(\sigma(\|y\|)^{2 i+1}\right)$ and $D_{y} \Psi=O\left(\sigma(\|y\|)^{2 i}\right)$. We can now use the same kind of bookkeeping as in the proof of lemma 2.2 to establish the result.. $]$

\subsection{Proof of lemma 3.2}

The proof of this lemma is a virtual duplication of the proof of lemma 2.3. This time we start with the Lyapunov function

$$
V=\sum_{i=1}^{n} \alpha_{i} \int_{0}^{y_{i}} \sigma^{2(n-i)+1}(s) d s
$$

where the $\alpha_{i}$ 's will be specified. The derivative along the trajectories of $(42)$ is given by

$$
\begin{aligned}
\dot{V} & =\sum_{i=1}^{n} \alpha_{i} \sigma^{2(n-i)+1}\left(y_{i}\right)\left[\dot{f}_{i}(y)+\vec{f}_{i}(y, t)\right] \\
& \leq\left[\sum_{i=1}^{n} \alpha_{i} \sigma^{2(n-i)+1}\left(x_{i}\right) A_{i} \psi(y)\right]+\gamma \sigma(\|y\|)^{2(n+1)+1}
\end{aligned}
$$

where $A_{i}$ is the $i$ th row of the matrix $A$ and $\gamma$ is a constant that depends on $\alpha_{i}, \beta_{i}$ for $i=1, \ldots, n$. We claim that the $\alpha_{i}$ 's can be chosen such that

$$
\left.\mathcal{S}(y) \equiv \sum_{i=1}^{n} \alpha_{i} \sigma^{2(n-i)+1}\left(y_{i}\right) A_{i} \psi(y) \leq-\sigma(\| y\} \mid\right)^{2(n+1)}
$$

This will give

$$
\dot{V} \leq-(1-\gamma \sigma(\|y\|)) \sigma(\|y\|)^{2(n+1)}
$$

and hence global asymptotic stability of the origin for $\epsilon$ sufficiently small. To prove this claim we now follow the proof of lemma 2.3 , everywhere replacing $\|y\|^{k}$ by $\sigma(\|y\|)^{k}$ and $y_{i}^{k}$ by $\sigma\left(y_{i}\right)^{k}$. The only difficulty we have is that the equality (61) does not carry over. However, it is sufficient to have the inequality

$$
\sigma\left(y_{n}\right)^{2} \geq \sigma(\|y\|)^{2}-\sigma\left(y_{1}\right)^{2}-\ldots-\sigma\left(y_{n-1}\right)^{2}
$$

Completely squares and judiciously choosing the $\alpha_{i}$ 's again produces the result. $\square$

\subsection{Proof of lemma 3.3}

The proof of this lemma follows from the proof of lemma 3.2. We use the same Lyapunov function $V$ as in (70). From lemma 3.2 and from the nature of the partial derivative of $V$ with respect to $y$, we have, for $\epsilon$ sufficiently small, that the derivative of $V$ along the trajectories of the perturbed system satisfies

$$
\dot{V} \leq-\left[1-\gamma \sigma(\|y\|)-\gamma \frac{\sigma(\|y\|)}{\sigma(\|y\|)^{2(n+1)}} \nu\right] \sigma(\|y\|)^{2(n+1)}
$$

Since we are simply trying to establish that $y$ is bounded we can as sume without loss of generality that $\delta^{2(n+1)}<\sigma(\|y\|)^{2(n+1)} \leq \epsilon^{2(n+1)}$ Therefore we see that if

$$
1-\gamma \epsilon-\gamma \frac{\epsilon}{\delta^{2(n+1)}} \nu>0
$$

then $\dot{V}<0$ for $|y|$ sufficiently large. Since $V$ is proper, this implies that $|y|$ is bounded. We see that, given $\epsilon$ such that $1-\gamma \epsilon>0,(76)$ is satisfied for all $\nu$ satisfying

$$
\nu<(1-\gamma \epsilon) \frac{\delta^{2(n+1)}}{\gamma \epsilon}
$$

口

\section{References}

[1] R. W. Brockett. Asymptotic stability and feedback stabilization. In R. W. Brockett, R. S. Millman, and H. J. Sussman, editors, Differential Geometric Control Theory, pages 181-191. Birkhauser, 1983

[2] J. Carr. Applications of Centre Manifold Theory. Springer Verlag, 1981

[3] J-M. Coron. Global asymptotic stabilization for controllable systems withour drift. Mathematics of Control, Signals, and Systems, 5(3), 1992.

[4] M. Grayson and R. Grossman. Models for free nilpotent Lie algebras. Technical Memo PAM-397, Center for Pure and Applied Mathematics, University of California, Berkeley, 1987. (to appear in J. Algebra)

[5] J. Guckenheimer and P. Holmes. Nonlinear Oscillations, Dynamical Systems, and Bifurcations of Vector Fields. Springer-Verlag, 1983.

[6] L. Gurvits and Z. X. Li. Smooth time-periodic feedback solutions for nonholonomic motion planning. Preprint, 1992.

[7] J.K. Hale. Ordinary Differential Equations. Wiley: New York, 1969.

[8] P. Jacobs, J-P. Laumond, and M. Taix. A complete iterative motion planner for a car-like robot. In Journees Geometrie Algorithmaque, INRIA, 1990.

[9] G. Lafferriere and H. J. Sussmann. Motion planning for controllable systems without drift. In IEEE International Conference on Robotics and Automation, pages $1148-1153,1991$

[10] J-P. Laumond and T. Siméon. Motion planning for a two degrees of freedom mobile robot with towing. In IEEE International Conference on Control and Applications, 1989.

[11] Z. Li and J. Canny. Motion of two rigid bodies with rolling constraint IEEE Transactions on Robotics and Automation, 6(1):62-71, 1990

[12] R. M. Murray and S. S. Sastry. Steering nonholonomic systems using sinusoids. In IEEE Control and Decision Conference, pages 2097-2101, 1990 .

[13] R. M. Murray and S. S. Sastry. Steering nonholonomic systems in chained form. In IEEE Control and Decision Conference, pages 1121-1126, 1991

[14] J-B. Pomet. Explicit design of time-varying stabilizing control laws for a class of controllable systems without drift. Systems and Control Letters, $18(2): 147-158,1992$.

[15] C. Samson and K. Ait-Abderrahim. Feedback stabilization of a nonholonomic wheeled mobile robot. In International Conference on Intelligent Robots and Systems (IROS), 1991

[16] E.D. Sontag. Remarks on stabilization and input-to-state stability. In Proceedings of the 28th Conference on Decision and Control, pages 13761378, December 1989

[17] H. J. Sussmann and W. Liu. Limits of highly oscillatory controls and the approximation of general paths by admissible trajectories. Technical Report SYCON-91-02, Rutgers Center for Systems and Control, 1991.

[18] D. Tilbury, J-P. Laumond, R. Murray, S. Sastry, and G. Walsh. Steering car-like systems with trailers using sinusoids. In IEEE International Conference on Robotics and Automation, 1992. 